\title{
Associação entre qualidade de vida e atividades desenvolvidas por idosos no Rio Grande do Sul
}

\section{Association between quality of life and activities developed by older adults from the Rio Grande do Sul state}

\author{
Margarete Diprat Trevisan', Patricia Morsch², Diene Gomes Colvara Lopes ${ }^{3}$, \\ Gabriela Guimarães de Oliveira ${ }^{4}$, Ângelo José Gonçalves Bós ${ }^{5}$
}

1 Bacharel em Fisioterapia. Doutoranda em Gerontologia Biomédica. Instituto de Geriatria e Gerontologia-PUCRS, Porto Alegre, RS, Brasil.<margadiprat@hotmail.com>
2 Bacharel em Fisioterapia. Doutora em Gerontologia Biomédica. Instituto de Geriatria e Gerontologia - PUCRS, Porto Alegre, RS, Brasil.<patriciamorsch@hotmail.com>
${ }^{3}$ Educação Física. Doutoranda em Gerontologia Biomédica. Instituto de Geriatria e Gerontologia - PUCRS, Porto Alegre, RS, Brasil.<dienegcl@hotmail.com>
${ }^{4}$ Bacharel em Fisioterapia. Mestranda em Gerontologia Biomédica. Instituto de Geriatria e Gerontologia-PUCRS, Porto Alegre, RS, Brasil.<oliveira_gabriela@hotmail.com>
${ }^{5}$ Medicina. PhD em Medicina, ênfase em Saúde Comunitária. Instituto de Geriatria e Gerontologia - PUCRS, Porto Alegre, RS, Brasil. < angelo.bos@pucrs.br>

Pesquisa original financiada pela Secretaria do Estado do Rio Grande do Sul e Escola de Saúde Pública do Rio Grande do Sul.

\section{ARTICLE INFO}

\section{Article history}

Received: 09/06/2017

Accepted: 28/09/2017

Correspondent Author

Margarete Diprat Trevisan

Rua Souza Lobo 1356 ap. 202

91320-321 Porto Alegre, RS

<margadiprat@hotmail.com>

(C) 2017 All rights reserved

Editors

Alfredo Cataldo Neto

Paula Engroff

\begin{abstract}
RESUMO
OBJETIVO: verificar a associação entre a qualidade de vida relacionada à saúde de idosos e a realização de diferentes atividades, ajustando os resultados para variáveis sociodemográficas e indicadores de saúde. MÉTODO: análise secundária do banco de dados do estudo denominado "Perfil dos Idosos do RS", uma pesquisa descritiva, transversal, de base domiciliar, com 7315 idosos comunitários, do Rio Grande do Sul. A qualidade de vida dos idosos nos domínios físico e mental foi avaliada pelo questionário SF-12, e as variáveis associadas, incluindo as atividades realizadas e indicadores de saúde, foram coletadas por autorrelato. Os testes $t$ de Student, ANOVA ou regressão linear simples, dependendo do tipo de variável, foram utilizados para a análise estatística simples, seguida por análise múltipla ajustada. RESULTADOS: Participaram do estudo 6052 idosos, 51,4\% do sexo feminino, principalmente na faixa etária entre 60-69 anos. A maioria das variáveis estudadas apresentou relação significativa com a qualidade de vida, em ambos os domínios, na análise simples. Porém, quando inseridas no modelo ajustado, apenas as variáveis, anos de estudo, número de morbidades e realização de atividades domésticas e autônomas se mantiveram estatisticamente significativas nos domínios físico e mental de qualidade de vida. CONCLUSÃO: O desenvolvimento de atividades é importante para a qualidade de vida dos idosos e, desta forma, a sociedade deve dispor de mais atividades estimulantes e inclusivas para esse grupo populacional, sendo importante levar em consideração a acessibilidade e diversidade, incluindo idosos com diferentes faixas etárias, nível educacional, hábitos e valores.
\end{abstract}

PALAVRAS-CHAVE: qualidade de vida; atividades humanas; envelhecimento; comorbidade.

\begin{abstract}
AIM: To verify the association between older adults' health-related quality of life and the performance of different activities, adjusting the results for sociodemographic variables and health indicators. METHOD: Secondary analysis of the "Older Adults' Profile in the RS state" database, a descriptive, cross-sectional, home-based study with 7315 community-dwelling older adults from the Rio Grande do Sul State. The older adults' quality of life, in the physical and mental domains, was assessed by the SF-12 questionnaire, and the associated variables, including activities and health indicators were selfreported measures. T Student test, ANOVA or simple linear regression, depending on the type of variable, were used for simple statistical analysis, followed by adjusted multiple analysis. RESULTS: Participated in the study 6052 older adults, $51.4 \%$ women, mainly in the $60-69$ age group. Most of the variables studied showed a significant association with quality of life, in both domains, in the simple analysis. However, in the adjusted model, only the variables years of study, number of morbidities and performance of domestic and autonomous activities remained statistically significant in the physical and mental domains of quality of life. CONCLUSION: The development of activities is important for the older adults' quality of life and, therefore, the society should have more stimulating and inclusive activities for this population group, taking into account accessibility and diversity, including elderly older adults from different age groups, educational status, habits and values.

KEYWORDS: quality of life; human activities; aging; comorbidity.
\end{abstract}




\section{INTRODUÇÃO}

O envelhecimento populacional é um fenômeno mundial que gera alterações demográficas, consequência do acentuado declínio na taxa de fertilidade e do aumento da expectativa de vida. Segundo a Organização das Nações Unidas (ONU), em 2050, o número de pessoas com mais de 60 anos de idade ou mais será cerca de três vezes maior do que o atual. Os idosos representarão aproximadamente um quinto da população mundial, o que gera uma grande preocupação a respeito da qualidade de vida durante o envelhecimento, pois esse processo pode ser acompanhado por declínio das capacidades funcionais, físicas e cognitivas ${ }^{1}$.

Juntamente com a transição demográfica, ocorre mundialmente a transição epidemiológica, caracterizada pela prevalência de doenças crônico-degenerativas, no lugar de doenças infecciosas, como causas de morbimortalidade. Viver mais pode significar o confronto de incapacidades, dependência, perda de papéis sociais, isolamento e depressão, culminando em redução da qualidade de vida. Essa sequência de acontecimentos gera um grande desafio para essa população e para os profissionais de saúde 2 .

A qualidade de vida (QV) é subjetiva e multifatorial, podendo ser definida como a percepção do indivíduo acerca da sua posição na vida no seu contexto cultural e no sistema de valores onde vive, em relação aos seus objetivos, expectativas, padrões e preocupações ${ }^{3}$, entre elas as relacionadas à saúde. A qualidade de vida relacionada à saúde (QVRS) é definida como "o valor atribuído à duração da vida, modificado pelos prejuízos, estados funcionais e oportunidades sociais que são influenciados por doença, dano, tratamento ou políticas de saúde" ${ }^{4}$. Estudos têm demonstrado que diferentes fatores podem influenciar a QVRS e o bem estar do idoso, como a prática de atividade física, que tem seu efeito positivo sobre a QV amplamente conhecido na literatura ${ }^{5-7}$ e o engajamento em atividades sociais até então menos estudadas ${ }^{8}$.

Entretanto, a importância das atividades sociais no idoso é conhecida, e por isso, é um dos pilares norteadores da Política de Envelhecimento Ativo proposta pela Organização Mundial da Saúde9. O engajamento social do idoso influencia diretamente a qualidade de vida, e até mesmo a percepção que ele tem de sua saúde ${ }^{10}$. O ambiente é fortemente associado à qualidade de vida para os idosos devido à sua relação com a interação social, ao envolvimento em atividades do cotidiano, à independência, à segurança e à proteção e bem-estar psicológico ${ }^{11}$.

A maior influência na qualidade de vida global dos idosos pode estar relacionada ao domínio físico, no entanto não podemos ignorar as alterações psicológicas e de inserção social características desta fase da vida. Deste modo, o objetivo deste estudo foi verificar a associação entre a qualidade de vida relacionada à saúde de idosos e a realização de diferentes tipos de atividades, ajustando os resultados para variáveis sociodemográficas e indicadores de saúde.

\section{MÉTODOS}

Este estudo é uma análise secundária do banco de dados do estudo denominado Perfil dos Idosos do RS (PIRS), realizado pelo Instituto de Geriatria e Gerontologia da Pontifícia Universidade Católica do Rio Grande do Sul (IGG-PUCRS) em parceria com a Escola de Saúde Pública do Rio Grande do Sul (ESP/RS). O PIRS foi realizado no período de 2010 a 2011, entrevistando 7315 idosos em 59 cidades do estado do Rio Grande do Sul/RS - Brasil, que objetivou traçar um perfil dos idosos do estado do RS, inspirado no Guia Global: Cidade Amiga do Idoso publicado em 2008 pela $\mathrm{OMS}^{12}$. Os métodos adotados no PIRS foram baseados na metodologia da pesquisa "Idosos do Rio Grande do Sul"13, onde cidades com mais de 25 mil habitantes foram aleatoriamente selecionadas. Os setores censitários de cada cidade receberam uma numeração sequencial com sorteio dos números. O número de setores sorteados por município foi relacionado ao número de idosos estimados, utilizando a mesma proporção de 3\% utilizada na pesquisa anterior. Foram entrevistados oito idosos por setor, sequencialmente um idoso do sexo masculino e uma idosa do sexo feminino. Primeiramente, o participante em potencial era identificado e, após a entrevista, reiniciava-se a identificação de um novo idoso a partir da $8^{\underline{a}}$ residência daquela mesma rua, ou seja, a cada idoso entrevistado, se contava oito casas e a partir dela se dava a busca de um novo participante.

O PIRS caracterizou-se, assim, por ser descritivo, populacional, de base domiciliar, de corte transversal, com coleta aleatória através de entrevista face-a-face. A população estudada foi composta por idosos, com idade acima de 60 anos, de ambos os sexos e residentes em domicílios urbanos e rurais no Estado do Rio Grande do Sul. Nos casos de idosos com déficit cognitivo, foram entrevistados os cuidadores ou familiares. Para verificar a habilidade cognitiva dos entrevistados foi aplicado o teste de recordação de três palavras. Neste, foi solicitado aos idosos que prestassem atenção nas palavras "irmão, chave e avião". O idoso deveria lembrar ao menos duas palavras. A metodologia utilizada no PIRS bem como a descrição detalhada do recrutamento dos participantes foi publicada recentemente pelos autores ${ }^{14}$. 
O desfecho utilizado foi a qualidade de vida dos idosos nos domínios físico e mental avaliado através do questionário SF-12. O Short Form-12 (SF-12) trata-se de um questionário utilizado para avaliar a qualidade de vida relacionada à saúde nos componentes físico e mental. A pontuação vai de 0 a 100 , onde o maior escore indica melhora no componente ${ }^{15}$. No presente estudo, foram excluídos indivíduos que apresentavam déficit cognitivo $(n=341)$ para reduzir o viés de resposta. Foram também excluídos os participantes que não responderam todas as questões do SF-12 $(n=922)$. As variáveis associadas foram as frequências com as quais os idosos participavam de atividades autônomas, cívicas, comunitárias, domésticas, remuneradas, sociais e voluntárias além de atividades como cuidar de outras pessoas e participar de grupos de idosos. Foram consideradas atividades autônomas aquelas de cunho remunerado onde o idoso não era empregado formal. As atividades cívicas foram representadas por atividades relacionadas à sua participação formal em eventos oficiais e de representação como votação, conselhos municipais e estaduais. Atividades comunitárias foram atividades desenvolvidas pela comunidade ou prefeitura com envolvimento e integração com outras faixas etárias. As atividades domésticas foram consideradas aquelas desenvolvidas dentro de casa, tanto nos afazeres diários como de manutenção do lar. As atividades remuneradas envolveram todas as atividades que produziam algum tipo de ganho pecuniário direto ou indireto. Atividades sociais envolveram a participação em festas, shows, bailes, almoços e eventos festivos, cinema, teatro, apresentações, bingos, jogos esportivos ou recreativos. As atividades voluntárias foram todas as atividades que não envolviam remuneração. Cuidar de outras pessoas incluía cuidar tanto de crianças quanto de pessoas mais velhas ou com algum grau de necessidades especiais. Já a participação em grupos de idosos correspondeu às atividades especificamente desenvolvidas nesses grupos. As variáveis de controle foram fatores sociodemográficos (idade em faixa etária, sexo e escolaridade medida pelo número de anos de estudo formal) e fatores relacionados à saúde (número de doenças crônicas que o idoso possuía).

Inicialmente foi realizada uma análise simples onde as médias dos escores de qualidade de vida relacionada à saúde foram calculadas, separadas nos dois componentes físico (SF-12 físico) e mental (SF12 mental), para os níveis das variáveis associadas e de controle e comparadas pelo teste t de Student ou ANOVA, exceto para as variáveis: anos de estudo e número de morbidades onde a regressão linear simples (usando o F estatístico) foi realizada para testar a associação positiva entre as variáveis. As variáveis significativas $(p<0,05)$ ou com indicativo de significância ( $\mathrm{p}$ entre 0,1 e 0,05), conforme Bós (2012) foram incluídas em modelos de regressão linear completos, um para cada componente do SF-12. Os modelos finais ajustados foram alcançados através da metodologia de retirada sistemática das variáveis menos significativas até que todas as variáveis remanescentes em cada modelo fossem significativas. Os dados foram analisados por meio de programa EPI INFO 3.5.3.

$\mathrm{O}$ projeto de pesquisa foi aprovado pelo Comitê de Ética da Escola de Saúde Pública do Rio Grande do Sul (Protocolo 481/09) e pelo Comitê de Ética em Pesquisa da Pontifícia Universidade Católica do Rio Grande do Sul (09/04931), cumprindo integralmente os princípios éticos contidos na declaração de Helsinki (2000), além do atendimento à legislação específica do Brasil. Os autores declaram não haver conflitos de interesses associados a esta pesquisa e seus resultados.

\section{RESULTADOS}

O número total de participantes no presente estudo foi 6052 , com leve predominância de mulheres $(51,4 \%)$ e a maioria dos entrevistados encontravam-se no grupo etário entre 60-69 anos (53,3\%). A análise descritiva completa e resultados referentes à análise simples da relação entre as variáveis independentes e o SF-12 físico encontram-se na Tabela 1.

Entre as variáveis demográficas observa-se que quanto maior a idade menor a média encontrada no SF-12 domínio físico e maior o desvio padrão, relação esta com significância estatística. Dentre a maioria das outras variáveis analisadas verifica-se que a participação social, sendo em atividades remuneradas ou comunitárias, apresenta relação positiva e significativa com a qualidade de vida no domínio físico, exceto as atividades domésticas que apresentou relação negativa e significante $(\mathrm{p}<0,001)$.

A análise simples do SF-12 mental apresenta padrão de resultados similares ao SF-12 físico, como demonstrado na Tabela 2 . A idade mostrou relação negativa e significativa com o SF-12 mental. Como no SF-12 físico, a participação social de maneira geral, apresentou relação positiva e significativa com o SF-12 mental. É importante salientar, porém que ao contrário do encontrado no SF-12 físico, as atividades autônomas obtiveram relação negativa com o SF-12 mental e as atividades domésticas relação positiva e significativa.

A Tabela 3 mostra os coeficientes de regressão linear para a qualidade de vida relacionada à saúde física e os fatores relacionados. A faixa etária 70-79 anos tinham em média 3,1\% pior qualidade de vida do que os participantes com 60 a 69 anos. Observamos também que quanto maior a faixa etária pior a qualidade de vida 
relacionada à saúde física. Na análise da escolaridade, constatamos que a cada ano de estudo correspondeu a uma melhora de 0,3\% no SF-12 físico. O número de morbidades, e realizar atividades domésticas foram negativamente associados à qualidade de vida. Realizar atividades autônomas e comunitárias foi positivamente associado à qualidade de vida enquanto que o gênero, realizar atividades remuneradas e atividades sociais não apresentaram associação. As atividades remuneradas que apresentaram relação significativa com o domínio físico do SF-12 na análise simples perderam significância estatística mostrando que o escore do SF-12 físico é dependente de outras variáveis.

A Tabela 4 mostra relação negativa na qualidade de vida relacionada à saúde mental quando relacionada às faixas etárias maiores, no entanto foi significativo apenas na comparação entre os indivíduos de 60 a 69 e os maiores que 90 anos. Assim como no componente físico, na qualidade de vida o número de morbidades e realizar atividades domésticas foram negativamente associadas à qualidade de vida no domínio mental. Entretanto, a atividade autônoma que foi positivamente associada à qualidade de vida no domínio físico, apresentou associação significativamente negativa relacionada ao domínio mental. Diferente do que foi encontrado no SF-12 físico, atividades comunitárias e história de quedas não apresentaram associação significativa. Da mesma forma, as atividades sociais que não foram associadas ao SF-12 físico, no domínio mental foram associadas positivamente.

Tabela 1. Análise descritiva e a relação entre o SF-12 físico e as variáveis independentes

\begin{tabular}{|c|c|c|c|c|}
\hline & N (\%) & Média & Devio Padrão & $\mathbf{p}$ \\
\hline $\begin{array}{l}\text { Sexo } \\
\text { Feminino } \\
\text { Masculino }\end{array}$ & $\begin{array}{l}3111(51,4 \%) \\
2941(48,6 \%)\end{array}$ & $\begin{array}{l}60,6 \\
61,3\end{array}$ & $\begin{array}{l}15,59 \\
15,30\end{array}$ & 0,1104 \\
\hline 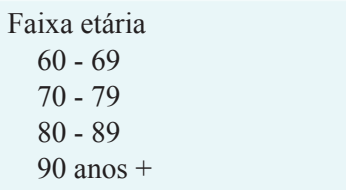 & $\begin{array}{c}3223(53,3 \%) \\
2092(34,6 \%) \\
687(11,4 \%) \\
50(0,8 \%)\end{array}$ & $\begin{array}{l}63,7 \\
58,9 \\
55,6 \\
45,4\end{array}$ & $\begin{array}{l}13,38 \\
16,24 \\
18,17 \\
22,51\end{array}$ & $<0,0001$ \\
\hline $\begin{array}{l}\text { Atividades autônomas } \\
\text { Não } \\
\text { Sim }\end{array}$ & $\begin{array}{c}5938(98.1 \%) \\
114(1.9 \%)\end{array}$ & $\begin{array}{l}60,9 \\
65,1\end{array}$ & $\begin{array}{l}15,49 \\
12,58\end{array}$ & 0,0035 \\
\hline $\begin{array}{l}\text { Atividades cívicas } \\
\text { Não } \\
\text { Sim }\end{array}$ & $\begin{array}{c}6000(99.1 \%) \\
52(0.9 \%)\end{array}$ & $\begin{array}{l}60,9 \\
62,4\end{array}$ & $\begin{array}{l}15,47 \\
13,34\end{array}$ & 0,5018 \\
\hline $\begin{array}{l}\text { Atividades comunitárias } \\
\text { Não } \\
\text { Sim }\end{array}$ & $\begin{array}{l}4965(82.0 \%) \\
1087(18.0 \%)\end{array}$ & $\begin{array}{l}60,3 \\
63,7\end{array}$ & $\begin{array}{l}15,78 \\
13,49\end{array}$ & $<0,0001$ \\
\hline $\begin{array}{l}\text { Atividades domésticas } \\
\text { Não } \\
\text { Sim }\end{array}$ & $\begin{array}{l}4401(72.7) \\
1651(27.3)\end{array}$ & $\begin{array}{l}61,6 \\
59,2\end{array}$ & $\begin{array}{l}15,08 \\
16,27\end{array}$ & $<0,0001$ \\
\hline $\begin{array}{l}\text { Atividade remunerada } \\
\text { Não } \\
\text { Sim }\end{array}$ & $\begin{array}{c}5671(93.7) \\
381(6.3)\end{array}$ & $\begin{array}{l}60,7 \\
64,9\end{array}$ & $\begin{array}{l}15,62 \\
11,87\end{array}$ & $<0,0001$ \\
\hline $\begin{array}{l}\text { Atividades sociais } \\
\text { Não } \\
\text { Sim }\end{array}$ & $\begin{array}{l}3527(58.3 \%) \\
2525(41.7 \%)\end{array}$ & $\begin{array}{l}59,9 \\
62,4\end{array}$ & $\begin{array}{l}16,37 \\
13,92\end{array}$ & 0,0001 \\
\hline $\begin{array}{l}\text { Atividades voluntárias } \\
\text { Não } \\
\text { Sim }\end{array}$ & $\begin{array}{c}5994(99.0 \%) \\
58(1.0 \%)\end{array}$ & $\begin{array}{l}60,9 \\
59,6\end{array}$ & $\begin{array}{l}15,44 \\
16,47\end{array}$ & 0,5234 \\
\hline $\begin{array}{l}\text { Cuida de outras pessoas } \\
\text { Não } \\
\text { Sim }\end{array}$ & $\begin{array}{c}6030(99.6 \%) \\
22(0.4 \%)\end{array}$ & $\begin{array}{l}60,9 \\
61,9\end{array}$ & $\begin{array}{l}15,45 \\
15,86\end{array}$ & 0,7600 \\
\hline $\begin{array}{l}\text { Participa de grupo de idosos } \\
\text { Não } \\
\text { Sim }\end{array}$ & $\begin{array}{c}5993(99.0 \%) \\
59(1.0 \%)\end{array}$ & $\begin{array}{l}61,0 \\
57,7\end{array}$ & $\begin{array}{l}15,44 \\
15,99\end{array}$ & 0,1078 \\
\hline & Média \pm DP & Coeficiente & F-estatístico & \\
\hline Anos de estudo & $4,9 \pm 3,72$ & 0,534 & 97,8738 & $<0,0001$ \\
\hline Número de morbidades & $1,3 \pm 1,43$ & $-2,533$ & 350,193 & $<0,0001$ \\
\hline
\end{tabular}


Tabela 2. Relação entre o SF-12 mental e as variáveis independentes

\begin{tabular}{|c|c|c|c|}
\hline & Média & Desvio Padrão & $\mathbf{p}$ \\
\hline $\begin{array}{l}\text { Sexo } \\
\qquad \text { Feminino } \\
\text { Masculino }\end{array}$ & $\begin{array}{l}60,2 \\
60,8\end{array}$ & $\begin{array}{l}10,66 \\
10,52\end{array}$ & 0,0196 \\
\hline 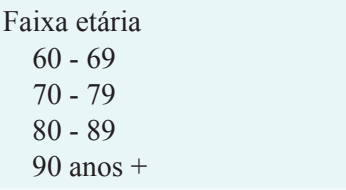 & $\begin{array}{l}60,9 \\
60,3 \\
59,4 \\
55,3\end{array}$ & $\begin{array}{l}9,58 \\
11,37 \\
12,01 \\
15,03\end{array}$ & $<0,0001$ \\
\hline $\begin{array}{l}\text { Atividades autônomas } \\
\text { Não } \\
\text { Sim }\end{array}$ & $\begin{array}{l}60,5 \\
57,1\end{array}$ & $\begin{array}{l}10,58 \\
11,01\end{array}$ & 0,0006 \\
\hline $\begin{array}{l}\text { Atividades cívicas } \\
\text { Não } \\
\text { Sim }\end{array}$ & $\begin{array}{l}60,5 \\
61,4\end{array}$ & $\begin{array}{c}10,61 \\
8,04\end{array}$ & 0,5296 \\
\hline $\begin{array}{l}\text { Atividades comunitárias } \\
\text { Não } \\
\text { Sim }\end{array}$ & $\begin{array}{l}60,4 \\
60,7\end{array}$ & $\begin{array}{l}10,98 \\
8,593\end{array}$ & 0,0004 \\
\hline $\begin{array}{l}\text { Atividades domésticas } \\
\text { Não } \\
\text { Sim }\end{array}$ & $\begin{array}{l}60,8 \\
59,5\end{array}$ & $\begin{array}{l}10,42 \\
10,97\end{array}$ & $<0,0001$ \\
\hline $\begin{array}{l}\text { Atividades remuneradas } \\
\text { Não } \\
\text { Sim }\end{array}$ & $\begin{array}{l}60,4 \\
61,3\end{array}$ & $\begin{array}{l}10,70 \\
8,800\end{array}$ & 0,1234 \\
\hline $\begin{array}{l}\text { Atividades sociais } \\
\text { Não } \\
\text { Sim }\end{array}$ & $\begin{array}{l}60,1 \\
61,0\end{array}$ & $\begin{array}{l}11,67 \\
8,846\end{array}$ & 0,0076 \\
\hline $\begin{array}{l}\text { Atividades voluntárias } \\
\text { Não } \\
\text { Sim }\end{array}$ & $\begin{array}{l}60,5 \\
59,8\end{array}$ & $\begin{array}{l}10,59 \\
10,87\end{array}$ & 0,6379 \\
\hline $\begin{array}{l}\text { Cuida de outras pessoas } \\
\text { Não } \\
\text { Sim }\end{array}$ & $\begin{array}{l}60,5 \\
61,8\end{array}$ & $\begin{array}{l}10,59 \\
11,92\end{array}$ & 0,5431 \\
\hline $\begin{array}{l}\text { Participa de grupo de idosos } \\
\text { Não } \\
\text { Sim }\end{array}$ & $\begin{array}{l}60,5 \\
59,8\end{array}$ & $\begin{array}{l}10,60 \\
10,39\end{array}$ & 0,6215 \\
\hline & Coeficiente & F-estatístico & \\
\hline Anos de estudo & 0,201 & 29,1186 & $<0,0001$ \\
\hline Número de morbidades & $-1,673$ & 323,3254 & $<0,0001$ \\
\hline
\end{tabular}

Tabela 3. Resultados das análises de regressão linear para SF-12 físico

\begin{tabular}{|c|c|c|c|c|}
\hline \multirow{2}{*}{ SF-12 físico } & \multicolumn{2}{|c|}{ Modelo completo } & \multicolumn{2}{|c|}{ Modelo final } \\
\hline & Coeficiente & $\mathbf{p}$ & Coeficiente & $\mathbf{p}$ \\
\hline $\begin{array}{l}\text { Sexo (referência feminino) } \\
\text { Masculino }\end{array}$ & $\begin{array}{c}1 \\
-0,050\end{array}$ & 0,8991 & & \\
\hline $\begin{array}{l}\text { Faixa etária (referência } 60 \text { a } 69 \text { anos) } \\
70-79 \text { anos } \\
80-89 \text { anos } \\
90 \text { anos ou mais }\end{array}$ & $\begin{array}{c}1 \\
-3,148 \\
-5,375 \\
-14,864\end{array}$ & $\begin{array}{l}<0,0001 \\
<0,0001 \\
<0,0001\end{array}$ & $\begin{array}{l}-3,204 \\
-5,440 \\
-14,978\end{array}$ & $\begin{array}{l}<0,0001 \\
<0,0001 \\
<0,0001\end{array}$ \\
\hline Anos de estudo & 0,263 & $<0,0001$ & 0,269 & $<0,0001$ \\
\hline Número de morbidades & $-2,081$ & $<0,0001$ & $-2,087$ & $<0,0001$ \\
\hline Atividades autônomas & 2,521 & 0,0700 & 2,478 & 0,0745 \\
\hline Atividades comunitárias & 3,443 & $<0,0001$ & 3,606 & $<0,0001$ \\
\hline Atividades domésticas & $-1,378$ & 0,0017 & $-1,397$ & 0,0011 \\
\hline Atividades remuneradas & 0,410 & 0,6081 & & \\
\hline Atividades sociais & 0,315 & 0,4577 & & \\
\hline
\end{tabular}


Tabela 4. Resultados das análises de regressão linear para a relação entre o SF-12 mental

\begin{tabular}{lcccc}
\hline \multirow{2}{*}{ SF-12 mental } & \multicolumn{2}{c}{ Modelo completo } & \multicolumn{2}{c}{ Modelo final } \\
\cline { 2 - 2 } $\begin{array}{l}\text { Sexo (referência feminino) } \\
\quad \text { Masculino }\end{array}$ & 0,250 & $\mathbf{p}$ & Coeficiente & p \\
Faixa etária (referência 60 a 69 anos) & & 0,3679 & & \\
$\quad 70-79$ anos & 0,070 & 0,8180 & 0,077 & 0,7965 \\
$\quad 80-89$ anos & $-0,418$ & 0,3559 & $-0,450$ & 0,3145 \\
$\quad$ 90 anos ou mais & $-3,909$ & 0,0081 & $-3,956$ & 0,0074 \\
Anos de estudo & 0,137 & 0,0003 & 0,141 & 0,0002 \\
Número de morbidades & $-1,590$ & $<0,0001$ & $-1,594$ & $<0,0001$ \\
Atividades autônomas & $-3,846$ & 0,0001 & $-3,798$ & 0,0001 \\
Atividades remuneradas & $-0,475$ & 0,4039 & & 0,0012 \\
Atividades comunitárias & 0,498 & 0,1927 & & 0,0044 \\
Atividades domésticas & $-0,933$ & 0,0029 & $-0,984$ & 0,791 \\
Atividades sociais & 0,641 & 0,0335 & & \\
\hline
\end{tabular}

\section{DISCUSSÃO}

Observamos que apesar das mulheres viverem em média cerca de quatro anos a mais do que os homens ${ }^{16}$, não significa que possuam melhor qualidade de vida. No estudo de Soares ${ }^{17}$, dentre as idosas estudadas, mais de $98 \%$ apresentaram pelo menos uma morbidade. Os resultados deste estudo, na análise simples, demonstraram que os homens obtiveram melhor qualidade de vida, este dado pode ter sido influenciado por outros fatores, pois quando foi realizada a análise ajustada este resultado perdeu a significância estatística. Em outro estudo, que buscou caracterizar o perfil, as condições de saúde e a qualidade de vida de octogenários que residem sozinhos em um município do norte do Rio Grande do Sul, encontrou que não houve diferença na qualidade de vida, com significância estatística, entre gêneros ${ }^{18}$.

Em relação à faixa etária quanto maior a idade menor a média encontrada no SF-12 domínio físico, assim como no domínio mental. Outro estudo demonstrou semelhança nos resultados encontrados, onde a qualidade de vida de idosos avaliados em três faixas etárias diferentes revelou que todas tendem a diminuir com o avançar da idade ${ }^{19}$. Andrade e Martins $^{20}$ na sua pesquisa com uma amostra onde a idade variou entre os 60 e 95 anos, demonstraram que os idosos com maior idade possuíam uma percepção de qualidade de vida menos satisfatória, com diferenças estatisticamente significativas.

Ainda considerando as variáveis sociodemográficas, em relação à escolaridade, constatamos que a cada ano de estudo correspondeu a uma melhora de 0,3\% no SF-12 físico. O que vai ao encontro do estudo de De Belvis et al. ${ }^{21}$ que investigaram a associação entre as relações sociais e de saúde relacionadas com a qualidade de vida de 33.744 idosos italianos residentes na comunidade, utilizando também o SF-12 e identificaram que maiores níveis de escolaridade associaram-se a maiores escores no domínio físico. Da mesma forma, $\mathrm{Cruz}^{22}$ que buscou identificar fatores associados à qualidade de vida em idosos não institucionalizados do município de São Paulo de 2006, por meio do SF-12 obtiveram associação entre o componente físico da qualidade de vida e à escolaridade.

Em nosso estudo, conforme esperado, verificouse que indivíduos com maior número de morbidades possuem menores índices de qualidade de vida tanto física, quanto mental. O mesmo foi encontrado em um estudo que buscou analisar a associação entre qualidade de vida e os fatores de risco para doenças crônicas não transmissíveis. O referido estudo constatou que os indivíduos que relataram hipertensão arterial, doenças reumatológicas, doenças vasculares, doenças cardíacas, doenças psiquiátricas ou diabetes mellitus obtiveram menores pontuações em diversas dimensões do questionário SF-36, demonstrando que os indivíduos portadores de variadas morbidades possuem menor qualidade de vida ${ }^{23}$. O mesmo resultado foi encontrado no estudo de Campolina et $\mathrm{al}^{24}$, que também utilizou o SF-36 para avaliar a qualidade de vida, sugerindo que o aumento do número de doenças crônicas impactou negativamente vários domínios da qualidade de vida. 
Além dos aspectos sociodemográficos e de saúde, as atividades realizadas pelo idoso também exercem influência sobre a qualidade de vida. A importância da atividade social e comunitária para os idosos é conhecida na literatura. Este tipo de atividade é de extrema importância para o idoso, pois pode trazer novas oportunidades e abrir espaço para interação social e manutenção da autonomia ${ }^{25}$. A participação social se mostra relevante ao fazer parte do Estatuto do Idoso, que procura facilitar o acesso de idosos a atividades de lazer, cultura, educação, entre outros ${ }^{26}$, indo ao encontro do que é recomendado na política de envelhecimento ativo da $\mathrm{OMS}^{9}$. Uma pesquisa que analisou a participação de idosos em grupos de convivência demonstrou que essa atividade proporcionou diversos benefícios, contribuindo para o exercício da cidadania, incentivando a autonomia e melhorando a qualidade de $v^{2}{ }^{27}$. No presente estudo, a participação social, incluindo festas, shows, bailes, almoços e eventos festivos, entre outros, apresentou influência positiva, com significância estatística apenas no domínio mental do SF-12. Ao contrário, a participação em atividades desenvolvidas na comunidade, apresentou relação significativa com o SF-12 apenas no domínio físico. Essa diferença pode ser explicada pelo tipo de atividade desenvolvida pelo idoso nas comunidades, as quais muitas vezes estão associadas à prática de atividade física, como dança, academia ao ar livre e grupos de caminhada. Outra explicação é a interpretação que o idoso fez da pergunta e do tipo de atividades que são disponibilizadas a eles, já que a resposta era autorrelatada.

Toscano e Oliveira ${ }^{6}$ compararam a qualidade de vida de idosos com distintos níveis de atividade física. Em seu estudo observaram que as atividades domésticas realizadas dentro e ao redor da habitação são as atividades que exigem o maior gasto energético dos idosos, por se tratar, normalmente, de atividades de intensidade moderada a vigorosa. Diante deste fato sugerimos que neste estudo a relação negativa e significativa das atividades domésticas no SF-12 físico deve-se a uma sobrecarga de cansaço apresentada por estes idosos.

Para Pondé e Caroso ${ }^{28}$ o prazer tem sido identificado como um fator fundamental para o bem-estar, abrindo a perspectiva de que não apenas as atividades de lazer, mas qualquer atividade relacionada com o prazer pode ter efeitos positivos na saúde mental. Tal argumento vai ao encontro deste estudo onde no SF-12 mental as atividades domésticas apresentaram relação positiva e significativa, o que sugere que estas atividades são prazerosas e benéficas para estes idosos.
Um estudo que objetivou avaliar a qualidade de vida de idosos cadastrados nas unidades de saúde da família de Foz do Iguaçu/PR verificou melhores índices de qualidade de vida nos idosos que exerciam atividade remunerada, tal resultado também foi encontrado neste estudo. Segundo Faller et $\mathrm{al}^{29}$, embora as atividades informais exijam maiores esforços e acarretem desgastes físico e mental, estas também permitem ao idoso manter-se ativo e contribuindo para a sociedade.

Observamos que desempenhar atividade autônoma foi positivamente relacionado à qualidade de vida física, no entanto no componente mental obteve associação negativa. Provavelmente isso se deve ao fato de que o trabalhador autônomo necessita ser mais ativo fisicamente para garantir seu rendimento, sem muita segurança financeira, carga horária máxima de trabalho, sem direito a férias ou qualquer outro benefício. Essas características de trabalho podem tornar o indivíduo mais saudável fisicamente, por ser mais ativo, por outro lado pode implicar em desgaste emocional em função da instabilidade característica deste ramo de atividade.

Algumas limitações do estudo devem ser consideradas. Primeiramente, o delineamento transversal, que não permite estabelecer relação de causalidade entre as variáveis. A condição de autorrelato das variáveis pode ser considerada um fator limitante, já que as respostas podem ser mais ou menos estimadas pelos participantes, como, por exemplo, na análise da qualidade de vida e realização de atividades específicas. Por outro lado, a condição de autorrelato também pode ser influenciada por déficits cognitivos, de atenção ou depressão. Para evitar esse viés, pacientes que não conseguiram lembrar-se de dois ou mais itens no teste de recordação de palavras foram excluídos. Além disso, poucos estudos para comparar os nossos achados foram encontrados na literatura a respeito da realização de atividades pelos idosos e a associação das mesmas com a qualidade vida.

\section{CONCLUSÃO}

Os poucos estudos encontrados sobre o tema em questão e os resultados encontrados demonstram a necessidade de ampliar atividades acessíveis aos idosos. Existem medidas que protegem o idoso nesse aspecto, porém são pouco incentivadas e respeitadas. Os resultados do presente estudo demonstram que as atividades realizadas pelo idoso são fundamentais para sua qualidade de vida, assim como fatores de saúde e sociodemográficos, que são amplamente conhecidos na literatura. 


\section{REFERÊNCIAS}

1. ONU. World Population Prospects: the 2010 revision. New York: Unites Nations; 2011.

2. Maués CR, et al. Avaliação da qualidade de vida: comparação entre idosos jovens e muito idosos. Rev Bras Clin Med. São Paulo. 2010;8(5);405-10.

3. The WHOQOL Group. The World Health Organization Quality of Life Assessment (WHOQOL): Position paper from the World Health Organization. Soc Sci Med. 1995; 41(10):1403-9.

4. Ebrahim S. Clinical and public health perspectives and applications of health-related quality of life measurement. Soc Sci Med. 1995;41:1383-94.

5. Gonçalves KC, et al. Comparação entre a percepção da qualidade de vida e o nível de aptidão física ee idosos praticantes de atividades aquáticas. Rev Bras Ciências da Saúde. 2014;12(39):35-43.

6. Toscano JJO, Oliveira ACC. Qualidade de vida em idosos com distintos níveis de atividade física. Rev Bras Med Esporte. 2009 maio/jun;15(3):169-73.

7. Matsudo SM, Matsudo VKR. Prescrição e benefícios da atividade física na terceira idade. Rev Bras Ciência e Movimento. 1992;6(4):19-30.

8. Huxhold $\mathrm{O}$, Miche M, Schüz B. Benefits of having friends in older ages: differential effects of informal social activities on well-being in middle-aged and older adults. Journ of Gerontology, Series B: Psychological Sciences and Social Sciences. 2013;69(3):366-75.

9. Organização Mundial da Saúde. Envelhecimento ativo: uma política de saúde, 2002. [acesso em 2014 nov 18]. Disponível em: http://bvsms.saude.gov.br/bvs/publicacoes/ envelhecimento_ativo.pdf

10. Pereira JK, Firmo JOA, Giacomin KC. Maneiras de pensar e de agir de idosos frente às questões relativas à funcionalidade/ incapacidade. Ciência \& Saúde Coletiva. 2014;19(8):3375-84.

11. Ribeiro, AP, et al. A influência das quedas na qualidade de vida de idosos. Ciência \& Saúde Coletiva. 2008;13(4): 1265-73.

12. Organização Mundial da Saúde. Guia Global: Cidade Amiga do Idoso. 2008. [acesso em 2012 jun 27]. Disponível em: http://www.who.int/ageing/GuiaAFCPortuguese.pdf

13. Rio Grande do Sul - Conselho Estadual do Idoso. Os idosos do Rio Grande do Sul: estudo multidimensional de suas condições de vida: relatório de pesquisa. Porto Alegre: CEI, 1997.

14. Pereira GN, et al. Fatores socioambientais associados à ocorrência de quedas em idosos. Cienc Saúde Colet. 2013; 18(12):3507-14.
15. Camelier AA. Avaliação da qualidade de vida relacionada à saúde em pacientes com DPOC: estudo de base populacional com o SF-12 na cidade de São Paulo-SP [Tese]. São Paulo (SP): Universidade Federal de São Paulo; 2004.

16. Beckert M, Irigaray TQ, Trentini CM. Qualidade de vida, cognição e desempenho nas funções executivas de idosos. Estudos de Psicologia Campinas. 2012 abr/jun;29(2): 155-62.

17. Soares $\mathrm{MBO}$, et al. Morbidades, capacidade funcional e qualidade de vida de mulheres idosas. Esc Anna Nery. 2010 out/dez;14(4):705-11.

18. Falcade BL, et al. Octogenários em residêcias unipessoais: enfoque sobre a qualidade de vida e condições de saúde. Rev Enfermagem, UFSM. 2011 set/dez;1(3):386-93.

19. Gonçalves LTH, et al. Convívio e cuidado familiar na quarta idade: qualidade de vida de idosos e seus cuidadores. Rev Bras Geriatr Gerontol. 2013;16(2):315-25.

20. Andrade A, Martins R. Funcionalidade Familiar e Qualidade de Vida dos Idosos. Millenium. 2011;40:185-99.

21. De Belvis AG, et al. Social relationships and HRQL: A cross-sectional survey among older Italian adults. BMC Public Health 2008 Oct;8(348):1-10.

22. Cruz KCT. Qualidade de vida relacionada à saúde dos idosos do Estudo SABE. [Tese] Faculdade de Saúde Pública Universidade Estadual de Campinas; 2012.

23. Campos MO, ET al. Impacto dos fatores de risco para doenças crônicas não transmissíveis na qualidade de vida. Ciência \& Saúde Coletiva. 2013;18(3): 873-82.

24. Campolina AG, Dini PS, Ciconelli RM. Impacto da doença crônica na qualidade de vida de idosos da comunidade em São Paulo. Ciência \& Saúde Coletiva. 2011;16(6): 2919-25.

25. Bulla LC, Soares ES, Kist RBB. Cidadania, pertencimento e participação social de idosos. Grupos Trocando Ideias e Matinê das Duas: Cine Comentado. Rev Ser Social. 2008 jul/dez;21:169-96.

26. BRASIL. Estatuto do Idoso. Brasília, 2003. [acesso em 2015 abr. 28]. Disponível em: http://www.planalto.gov.br/ccivil 03/ leis/2003/110.741.htm

27. Kist RBB; Bulla, LC. A participação em espaços coletivos e a autonomia dos idosos. Temporalis $2014 \mathrm{jul} / \mathrm{dez} ; 14(28)$ : 151-71.

28. Pondé MP, Caroso C. Lazer como fator de proteção da saúde mental. Rev Ciênc Méd. 2013 abr/jun;12(2):163-72.

29. Faller JW, et al. Qualidade de vida de idosos cadastrados na estratégia saúde da família de Foz do Iguaçu-PR. Esc Anna Nery. 2010 out-dez;14(4):803-10. 